\title{
Lucas Symbolic Formulae and Generating Functions for Chebyshev Polynomials
}

\author{
Do Tan $\mathrm{Si}^{1,2,3}$ \\ ${ }^{1}$ Physical Association, Ho Chi Minh City, Vietnam \\ ${ }^{2}$ ULB, Bruxelles, Belgium \\ ${ }^{3}$ UEM, Mons, Belgium \\ Email: tansi_do@yahoo.com
}

How to cite this paper: Si, D.T. (2021) Lucas Symbolic Formulae and Generating Functions for Chebyshev Polynomials. Journal of High Energy Physics, Gravitation and Cosmology, 7, 914-924.

https://doi.org/10.4236/jhepgc.2021.73052

Received: May 3, 2021

Accepted: June 22, 2021

Published: June 25, 2021

Copyright (c) 2021 by author(s) and Scientific Research Publishing Inc. This work is licensed under the Creative Commons Attribution International License (CC BY 4.0).

http://creativecommons.org/licenses/by/4.0/

\begin{abstract}
This work shows that each kind of Chebyshev polynomials may be calculated from a symbolic formula similar to the Lucas formula for Bernoulli polynomials. It exposes also a new approach for obtaining generating functions of them by operator calculus built from the derivative and the positional operators.
\end{abstract}

\section{Keywords}

Chebyshev Polynomials, Lucas Symbolic Formula, Generating Functions by Operator Calculus

\section{Introduction}

The Chebyshev polynomials of the first kind $T_{n}(x)$ and second kind $U_{n}(x)$, very important in approximation theory among others, were defined by Tchebychef in the year 1899 and may be consulted on the net, for example in Wikipedia or in an important work of Markov, Andrey Andreevich, Sonin [1]. Although various properties of their area abundantly studied by classical method, we would like to find a formula for calculating each of them not by cumbersome recurrence but by a symbolic formula suggested by the symbolic Lucas formula for calculating Bernoulli polynomials, say $B_{n}(x)=:(B+x)^{n} \quad$ [2]. This is possible because Chebyshev polynomials may be put into the form of a special operator applying on monomials [3].

\section{Definitions of Chebyshev Polynomials of the $1^{\text {st }}$ Kind}

\subsection{By Trigonometric Functions}

The Chebyshev polynomials of the first kind $T_{n}(x)$ [1] [4] may be defined by 
many approaches, one of these is by the formula

$$
T_{n}(\cos (x))=\cos (n x)
$$

or,

$$
T_{n}(x)=\cos (n \arccos (x))=\operatorname{Re} \mathrm{e}^{i n \arccos (x)}=\operatorname{Re}\left(x+i \sqrt{1-x^{2}}\right)^{n}
$$

For familiarization with trigonometric functions we cite the following properties:

$$
\begin{aligned}
& 2 T_{n}(x) T_{m}(x)=2 \operatorname{Re}^{i n \arccos (x)} \operatorname{Re}^{i m \arccos (x)} \\
& =2(\cos (n \arccos (x)) \cos (m \arccos (x))-\sin (n \arccos (x)) \sin (m \arccos (x))) \\
& =\cos ((n+m) \arccos (x))+\cos ((n-m) \arccos (x)) \\
& =T_{n+m}(x)+T_{n-m}(x)
\end{aligned}
$$

For examples:

$$
\begin{aligned}
& T_{n+1}(x)=2 x T_{n}(x)-T_{n-1}(x) \\
& T_{2 n}(x)=2 T_{n}^{2}(x)-1 \\
& T_{4}(x)=2 T_{2}^{2}(x)-1=2\left(2 x^{2}-1\right)^{2}-1=8 x^{4}-8 x^{2}+1 \\
& \text { ○ } T_{n}\left(T_{m}(\cos \theta)\right)=T_{n}(\cos (m \theta))=\cos (n m \theta)=T_{n m}(\cos \theta) \\
& T_{n}\left(T_{m}(x)\right)=T_{n m}(x) \\
& T_{n}^{\prime}(x)=n \frac{-1}{\sin (\arccos (x))} \operatorname{Re} i \mathrm{e}^{i n \arccos (x)}=n \frac{\sin (n \arccos (x))}{\sin (\arccos (x))} \\
& =2 \cos (n \arccos (x))=2 T_{n}(\cos (\arccos (x)))=2 T_{n}(x) \\
& T_{n}\left(\frac{x+x^{-1}}{2}\right)=\left(\frac{x^{n}+x^{-n}}{2}\right)
\end{aligned}
$$

\subsection{By Hyper-Differential Operators}

Another interesting definition $T_{n}(x)$ is that they are related to the Gegenbauer polynomials by the relation [4].

$$
T_{n}(x)=: n !{ }_{0} F_{1}\left(-; \frac{1}{1 / 2} ;-\frac{B^{2}}{4}\right) \frac{x^{n}}{n !}
$$


where $\hat{B}$ is the operator defined from the derivative operator $D_{x}$ and the Eckaert operator $\hat{X}$ which means "multiplied with the argument" and verifying the commutation relation $\left[D_{x}, \hat{X}\right] \equiv I$.

$$
\begin{gathered}
\hat{B} \equiv\left(1-\hat{X}^{2}\right)^{\frac{1}{2}} D_{x} \\
\hat{B}^{k} \equiv: \hat{B}_{k} \equiv\left(1-\hat{X}^{2}\right)^{\frac{k}{2}} D_{x}^{k}
\end{gathered}
$$

Concretely we have the symbolic definition,

$$
T_{n}(x)=\sum_{k=0}^{\infty}(-1)^{k} \frac{1}{(2 k) !}\left(1-x^{2}\right)^{k} D_{x}^{2 k} x^{n}=: \cos \hat{B} x^{n}
$$

The formula (13) is very convenient for obtaining the following generating functions of $T_{n}(x)$ which were proven by Cesarano by another approach [5].

Firstly,

$$
\begin{aligned}
\sum_{n=0}^{\infty} T_{n}(x) \frac{t^{n}}{n !} & =: \cos \hat{B} \mathrm{e}^{t x}=\sum_{k=0}^{\infty}(-1)^{k} \frac{1}{(2 k) !}\left(1-x^{2}\right)^{k} t^{2 k} \mathrm{e}^{t x} \\
& =\cos \left(t \sqrt{1-x^{2}}\right) \mathrm{e}^{t x},|x|<1
\end{aligned}
$$

Secondly because,

$$
D_{x}^{2 k} \frac{1}{x}=\frac{(2 k) !}{x^{2 k+1}}
$$

we may write for $|x t|<1$,

$$
\begin{aligned}
\sum_{n=0}^{\infty} T_{n}(x) t^{n} & =: \cos B \frac{1}{1-x t}=\sum_{k=0}^{\infty} \frac{1}{(2 k) !}\left(x^{2}-1\right)^{k} D_{x}^{2 k} \frac{1}{1-x t} \\
& =\frac{1}{1-x t} \sum_{k=0}^{\infty}\left(\frac{\left(x^{2}-1\right) t^{2}}{(1-x t)^{2}}\right)^{k}=\frac{1}{1-x t}\left(\frac{(1-x t)^{2}}{(1-x t)^{2}-\left(x^{2}-1\right) t^{2}}\right) \\
& =\frac{1-x t}{1-2 x t+t^{2}}
\end{aligned}
$$

Examples:

For $t=1, x=\cos \theta$ we have:

$$
\cos \theta+\cos 2 \theta+\cdots+\cos n \theta+\cdots=\frac{1}{2}
$$

which gives the formula unwillingly stated without proof by Euler [6].

$$
1-1+1-1+\cdots=\frac{1}{2}
$$

Thirdly because for $-1<x<1$,

$$
\begin{gathered}
\sum_{n=1}^{\infty} \frac{x^{n}}{n}=\sum_{n=0}^{\infty} \int x^{n}=\int \frac{1}{1-x}=\ln \frac{1}{1-x} \\
D_{x} \ln \frac{1}{1-x}=-D_{x} \ln (1-x)=\frac{1}{1-x} \\
D_{x}^{2 k} \ln \frac{1}{1-x}=(2 k-1) !(1-x)^{-2 k}, \quad k>0
\end{gathered}
$$


we may write for $|x|<1,|x t| \leq 1$,

$$
\begin{aligned}
\sum_{n=1}^{\infty} T_{n}(x) \frac{t^{n}}{n} & =: \cos \hat{B} \sum_{n=1}^{\infty} \frac{(x t)^{n}}{n}=: \cos \hat{B}\left(\ln \frac{1}{1-x t}\right)=\sum_{k=0}^{\infty} \frac{\left(x^{2}-1\right)^{k}}{(2 k) !} D_{x}^{2 k}\left(\ln \frac{1}{1-x t}\right) \\
& =\ln \frac{1}{1-x t}+\sum_{k=1}^{\infty} \frac{\left(x^{2} t^{2}-t^{2}\right)^{k}}{(2 k) !}(2 k-1) !(1-x t)^{-2 k} \\
& =\ln \frac{1}{1-x t}+\frac{1}{2} \sum_{k=1}^{\infty} \frac{1}{k}\left(\frac{x^{2} t^{2}-t^{2}}{(1-x t)^{2}}\right)^{k} \\
& =\ln \frac{1}{1-x t}+\frac{1}{2} \ln \frac{1}{1+\frac{t^{2}-x^{2} t^{2}}{(1-x t)^{2}}}=\ln \frac{1}{1-x t}+\frac{1}{2} \ln \frac{(1-x t)^{2}}{1-2 x t+t^{2}} \\
& =\ln \frac{1}{1-x t}+\ln (1-x t)+\frac{1}{2} \ln \frac{1}{1-2 x t+t^{2}}=\ln \frac{1}{\sqrt{1-2 x t+t^{2}}}
\end{aligned}
$$

As examples, for $|x|<1, t=1$ we have successively,

$$
\frac{T_{1}(x)}{1}+\frac{T_{2}(x)}{2}+\cdots+\frac{T_{n}(x)}{n}+\cdots=-\frac{1}{2} \ln (1-x)
$$

i.e.

$$
\begin{gathered}
\frac{\cos (x)}{1}+\frac{\cos (2 x)}{2}+\cdots+\frac{\cos (n x)}{n}+\cdots=-\frac{1}{2} \ln (1-\cos (x)) \\
\frac{\cos (\pi / 2)}{1}+\frac{\cos (\pi)}{2}+\cdots+\frac{\cos (n \pi / 2)}{n}+\cdots=-\frac{1}{2} \ln (1-\cos (\pi / 2)) \\
-\frac{1}{2}+\frac{1}{4}-\frac{1}{6}+\frac{1}{8}+\cdots=-\frac{1}{2} \ln 1=0
\end{gathered}
$$

Consequently by derivation of (20) then putting $x=\frac{\pi}{2}$ we get:

$$
\sin (x)+\sin (2 x)+\cdots+\sin (n x)+\cdots=\frac{1}{2} \frac{\sin (x)}{1-\cos (x)}
$$

and the Euler's assertion [6],

$$
1+0-1+0+\cdots+\sin \left(n \frac{\pi}{2}\right)+\cdots=\frac{1}{2} \frac{\sin (\pi / 2)}{1-\cos (\pi / 2)}=\frac{1}{2}
$$

Lastly because,

$$
\begin{gathered}
\int \ln x=x \ln x-1 \\
\int \ln (1-x)=-(1-x) \ln (1-x)-1 \\
D_{x} \ln \frac{1}{1-x}=-D_{x} \ln (1-x)=\frac{1}{1-x} \\
D_{x}((x-1) \ln (1-x)-1)=D_{x}((x-1) \ln (1-x)) \\
=-(x-1) \frac{1}{1-x}-\ln (1-x)=1+\ln \frac{1}{1-x}
\end{gathered}
$$




$$
\begin{gathered}
D_{x}^{2}((x-1) \ln (1-x)-1)=D_{x}\left(\ln \left(\frac{1}{1-x}\right)+1\right)=D_{x}\left(\ln \left(\frac{1}{1-x}\right)\right)=\frac{1}{1-x} \\
D_{x}^{2 k}((x-1) \ln (1-x)-1)=(2 k-1) !\left(\frac{1}{1-x}\right)^{2 k-1}
\end{gathered}
$$

we have,

$$
\sum_{n=1}^{\infty} \frac{x^{n}}{n^{2}}=\int \sum_{n=1}^{\infty} \frac{x^{n-1}}{n}=\int \ln \left(\frac{1}{1-x}\right)=(1-x) \ln \frac{1}{1-x}-1
$$

and,

$$
\begin{aligned}
\sum_{n=1}^{\infty} T_{n}(x) \frac{t^{n}}{n^{2}} & =: \cos \hat{B} \sum_{n=1}^{\infty} \frac{(x t)^{n}}{n^{2}}=: \cos \hat{B}(1-x t)\left(\ln \frac{1}{1-x t}\right) \\
& =: \sum_{k=0}^{\infty} \frac{\left(x^{2}-1\right)^{k}}{(2 k) !} D_{x}^{2 k}(1-x t)\left(\ln \frac{1}{1-x t}\right) \\
& =\sum_{k=0}^{\infty} \frac{\left(x^{2} t^{2}-t^{2}\right)^{k}}{(2 k) !}(2 k-1) !\left(\frac{1}{1-x t}\right)^{2 k-1} \\
& =\frac{1}{1-x t} \frac{1}{2} \ln \frac{1}{1-\frac{x^{2} t^{2}-t^{2}}{(1-x t)^{2}}} \\
& =\frac{1}{1-x t}\left(\ln (1-x t)+\ln \frac{1}{\sqrt{1-2 x t+t^{2}}}\right)
\end{aligned}
$$

\section{Obtaining Lucas Formula for Chebyshev Polynomials of the $1^{\text {st }}$ Kind}

\subsection{Current Method}

Until now the polynomials $T_{n}(x)$ may be calculated by the formulae deduced from (2), (3),

$$
\begin{gathered}
T_{n}(x)=\operatorname{Re}\left(x+i \sqrt{1-x^{2}}\right)^{n} \\
T_{n+1}(x)=2 x T_{n}(x)-T_{n-1}(x) \\
T_{2 n}(x)=2 T_{n}^{2}(x)-1
\end{gathered}
$$

Nevertheless remarking that the Bernoulli polynomials may be calculated advantageously by the Lucas symbolic formula [2].

$$
B_{n}(x)=:(B+x)^{n}
$$

where the undefined coefficients $B^{k}$ are to be replaced with well-defined Bernoulli numbers $B_{k}$ [7], for example,

$$
B_{3}(x)=:(B+x)^{3}=: B_{0} x^{3}+3 B_{1} x^{2}+3 B_{2} x+B_{3}
$$

we will hereafter try to obtain a similar symbolic formula for Chebyshev polynomials. 


\subsection{Symbolic Formula for Calculating $T_{n}(x)$}

Consider the symbolic formula (13):

$$
T_{n}(x)=: \cos \hat{B} x^{n}=\sum_{k=0}^{\infty}(-1)^{k} \frac{1}{(2 k) !}\left(1-x^{2}\right)^{k} D_{x}^{2 k} x^{n}
$$

Let,

$$
u=x+y
$$

We have:

$$
\partial_{x}=\frac{\mathrm{d} u}{\mathrm{~d} x} \partial_{u}=\frac{\mathrm{d} u}{\mathrm{~d} y} \partial_{u}=\partial_{y}=\partial_{u}
$$

so that,

$$
\begin{aligned}
T_{n}(x+y) & =\sum_{k=0}^{\infty} \frac{1}{(2 k) !}\left((x+y)^{2}-1\right)^{k} \partial_{y}^{2 k}(x+y)^{n} \\
& =\sum_{k=0}^{\infty} \frac{1}{(2 k) !}\left((x+y)^{2}-1\right)^{k} \partial_{y}^{2 k} \sum_{l=0}^{n}\left(\begin{array}{l}
n \\
l
\end{array}\right) x^{l} y^{n-l}
\end{aligned}
$$

For $y=0$ we get:

$$
\begin{gathered}
T_{n}(x)=\sum_{k=0}^{\infty} \frac{1}{(2 k) !}\left(x^{2}-1\right)^{k} \partial_{y}^{2 k} \sum_{l=0}^{n}\left(\begin{array}{c}
n \\
l
\end{array}\right) y^{l} x^{n-l}, l=2 k \leq n \\
=\sum_{k=0}^{\infty} \frac{1}{(2 k) !}\left(x^{2}-1\right)^{k}(2 k) !\left(\begin{array}{c}
n \\
2 k
\end{array}\right) x^{n-2 k} \\
T_{n}(x)=\sum_{k=0}^{\left[\frac{n}{2}\right]}\left(x^{2}-1\right)^{k}\left(\begin{array}{c}
n \\
2 k
\end{array}\right) x^{n-2 k}
\end{gathered}
$$

In the above formula $k$ must be pair for $T_{n}(x)$ to have the parity of $n$. Finally defining,

$$
\begin{gathered}
C_{2 k+1}(x)=0 \\
C_{2 k}(x)=\left(x^{2}-1\right)^{k}
\end{gathered}
$$

we obtain the symbolic formula for calculating $T_{n}(x)$,

$$
T_{n}(x)=:(C+x)^{n}
$$

where undefined terms $C^{k}$ are to be replaced with $C_{k}(x)$.

For examples:

$$
\begin{gathered}
T_{0}(x)=1 \\
T_{1}(x)=C_{0} x^{1}+C_{1} x^{0}=x \\
T_{2}(x)=C_{0} x^{2}+C_{2} x^{0}=x^{2}+\left(x^{2}-1\right) \\
T_{3}(x)=C_{0} x^{3}+3 C_{2} x=x^{3}+3\left(x^{2}-1\right) x=4 x^{3}-3 x \\
T_{4}(x)=C_{0} x^{4}+6 C_{2} x^{2}+C_{4}=x^{4}+6\left(x^{2}-1\right) x^{2}+\left(x^{2}-1\right)^{2}=2^{3} x^{4}-8 x^{2}+1
\end{gathered}
$$

i.e. 


$$
\begin{gathered}
T_{2}(\cos x)=\cos 2 x=2 \cos ^{2} x-1 \\
T_{3}(\cos x)=\cos 3 x=4 \cos ^{3} x-3 \cos x \\
T_{4}(\cos x)=8 \cos ^{4}(x)-8 \cos ^{2}(x)+1 \\
=\cos 4 x=\cos ^{4}(x)+6 \sin ^{2}(x) \cos ^{2}(x)+\sin ^{4}(x)
\end{gathered}
$$

As consequence, because,

$$
\cos 2 x=2 \cos ^{2} x-1
$$

we get a maybe new formula for number theory,

$$
\begin{gathered}
2^{2 n-1}=\left(\begin{array}{c}
2 n \\
0
\end{array}\right)+\left(\begin{array}{c}
2 n \\
2
\end{array}\right)+\cdots+\left(\begin{array}{c}
2 n \\
2 n
\end{array}\right) \\
2^{2 n}=\left(\begin{array}{c}
2 n+1 \\
0
\end{array}\right)+\left(\begin{array}{c}
2 n+1 \\
2
\end{array}\right)+\cdots+\left(\begin{array}{c}
2 n+1 \\
2 n
\end{array}\right)
\end{gathered}
$$

\section{The Chebyshev Polynomials of the Second Kind}

\subsection{Definitions and Symbolic Formula for Calculation}

The Chebyshev polynomials of the second kind $U_{n}(x)$ may be defined trigonometrically [1] [4] by:

$$
U_{n}(\cos x)=\frac{\sin ((n+1) x)}{\sin (x)}
$$

or via the operator $\hat{B}$ by [3],

$$
U_{n}(x)=:(n+1) \frac{\sin \hat{B}}{\hat{B}} x^{n}
$$

In fact from (36) and for $-1<x<1$ we get:

$$
\begin{aligned}
U_{n}(x) & =(n+1) \sum_{k=0}^{\infty}(-1)^{k} \frac{\left(1-x^{2}\right)^{k} D_{x}^{2 k}}{(2 k+1) !} x^{n} \\
& =\sum_{k=0}^{\left[\frac{n}{2}\right]}(-1)^{k} \frac{\left(1-x^{2}\right)^{k}}{(2 k+1) !} x^{n-2 k} \frac{(n+1) !}{(n-2 k) !} \\
& =\frac{1}{i}\left(1-x^{2}\right)^{-\frac{1}{2}} \sum_{k=0}^{\left[\frac{n}{2}\right]} i^{2 k+1}\left(\begin{array}{c}
n+1 \\
2 k+1
\end{array}\right){\sqrt{1-x^{2}}}^{2 k+1} x^{n+1-2 k-1} \\
& =\frac{1}{\sqrt{1-x^{2}}} \operatorname{Im}\left(x+i \sqrt{1-x^{2}}\right)^{n+1}=\frac{1}{\sqrt{1-x^{2}}} \operatorname{Ime}^{i(n+1) \arccos (x)} \\
& =\frac{1}{\sqrt{1-x^{2}}} \operatorname{Im} \mathrm{e}^{i(n+1) \arccos (x)}
\end{aligned}
$$

i.e.,

$$
U_{n}(\cos \theta)=\frac{1}{\sin \theta} \operatorname{Im} \mathrm{e}^{i(n+1) \theta}=\frac{1}{\sin \theta} \sin ((n+1) \theta)
$$

\subsection{Generating Functions}

Utilizing the property, 


$$
\partial_{t} \hat{T} t^{n}=\partial_{t} t^{n+1}=(n+1) t^{n}
$$

we get the generating functions for $|x|<1$,

$$
\begin{aligned}
\sum_{n=0}^{\infty} U_{n}(x) \frac{t^{n}}{n !} & =: \frac{\sin B}{B} \sum_{n=0}^{\infty}(n+1) \frac{x^{n} t^{n}}{n !}=: \partial_{t} \hat{T} \frac{\sin B}{B} \sum_{n=0}^{\infty} \frac{x^{n} t^{n}}{n !}=: \partial_{t} \hat{T} \frac{\sin B}{B} \mathrm{e}^{x t} \\
& =: \partial_{t} \hat{T} \sum_{k=0}^{\infty}(-1)^{k} \frac{B^{2 k}}{(2 k+1) !} \mathrm{e}^{x t} \\
& =: \partial_{t} \hat{T} \sum_{k=0}^{\infty}(-1)^{k} \frac{1}{(2 k+1) !}\left(1-x^{2}\right)^{k} D_{x}^{2 k} \mathrm{e}^{x t} \\
& =\partial_{t} \frac{\sin t \sqrt{1-x^{2}}}{\sqrt{1-x^{2}}} \mathrm{e}^{x t} \\
& =\left(\frac{x}{\sqrt{1-x^{2}}} \sin \left(t \sqrt{1-x^{2}}\right)+\cos \left(t \sqrt{1-x^{2}}\right)\right) \mathrm{e}^{x t}
\end{aligned}
$$

and for $|x|<1,|x t|<1$,

$$
\begin{aligned}
\sum_{n=0}^{\infty} U_{n}(x) t^{n} & =: \frac{\sin B}{B} \sum_{n=0}^{\infty}(n+1) x^{n} t^{n}=: \frac{\sin B}{B} \partial_{t} \hat{T} \sum_{n=0}^{\infty} x^{n} t^{n} \\
& =\sum_{k=0}^{\infty}(-1)^{k} \frac{\left(1-x^{2}\right)^{k}}{(2 k+1) !} D_{x}^{2 k} \frac{1}{1-x t}=\sum_{k=0}^{\infty}(-1)^{k} \frac{\left(1-x^{2}\right)^{k}}{(2 k+1) !} D_{x}^{2 k} \frac{1}{(1-x t)^{2}} \\
& =\sum_{k=0}^{\infty}(-1)^{k} \frac{\left(t^{2}-x^{2} t^{2}\right)^{k}}{(1-x t)^{2+2 k}}=(1-x t)^{-2} \frac{1}{1+\frac{t^{2}-x^{2} t^{2}}{(1-x t)^{2}}}=\frac{1}{1-2 x t+t^{2}}
\end{aligned}
$$

From (40) and with $t=1$ we get:

$$
U_{0}(x)+U_{1}(x)+\cdots+U_{n}(x)+\cdots=\frac{1}{2} \frac{1}{1-x}, \quad|x|<1
$$

which following (37) proves the famous Euler's assertion) [6],

$$
1-1+1-1+\cdots=\frac{1}{2}
$$

\subsection{Interrelations between $T_{n}(x)$ and $U_{n}(x)$}

From (2) and (37),

$$
\begin{gathered}
T_{n}(x)=\operatorname{Re} \mathrm{e}^{i n \arccos (x)}=\operatorname{Re}\left(x+i \sqrt{1-x^{2}}\right)^{n} \\
U_{n}(x)=\frac{1}{\sqrt{1-x^{2}}} \operatorname{Im} \mathrm{e}^{i(n+1) \arccos (x)}
\end{gathered}
$$

we get:

$$
\begin{aligned}
T_{n+1}(x) & =\operatorname{Re}^{i n \arccos (x)} \mathrm{e}^{i \arccos (x)} \\
& =\operatorname{Re} \mathrm{e}^{i n \arccos (x)} \cos (\arccos (x))-\operatorname{Im} \mathrm{e}^{i \arccos (x)} \sin (\arccos (x)) \\
& =x T_{n}(x)-\operatorname{Im} \mathrm{e}^{i n \arccos (x)} \sin (\arccos (x)) \\
& =x T_{n}(x)-\sqrt{1-x^{2}} U_{n-1}(x) \sqrt{1-x^{2}}
\end{aligned}
$$


i.e.,

$$
T_{n+1}(x)=x T_{n}(x)-\left(1-x^{2}\right) U_{n-1}(x)
$$

Similarly we have 1

$$
\begin{aligned}
U_{n}(x) & =\frac{1}{\sqrt{1-x^{2}}} \operatorname{Im} \mathrm{e}^{i n \arccos (x)} \mathrm{e}^{\operatorname{iarccos}(x)} \\
& =\frac{1}{\sqrt{1-x^{2}}}\left(\operatorname{Re} \mathrm{e}^{i n \arccos (x)} \operatorname{Im} \mathrm{e}^{i \arccos (x)}+\operatorname{Im} \mathrm{e}^{i n \arccos (x)} \operatorname{Re}^{i \arccos (x)}\right) \\
& =T_{n}(x)+x U_{n-1}(x)
\end{aligned}
$$

and,

$$
\begin{gathered}
T_{n}(x)+i \sqrt{1-x^{2}} U_{n-1}(x)=\left(x+i \sqrt{1-x^{2}}\right)^{n} \\
T_{n}(\cos (\theta))+i \sin (\theta) U_{n-1}(\cos (\theta))=\mathrm{e}^{i n \cos (\theta)}
\end{gathered}
$$

An interesting relation comes from (2), (37) is,

$$
\begin{gathered}
D_{x} T_{n}(x)=n(\arccos (x))^{\prime} \operatorname{Re} i \mathrm{e}^{i n \arccos (x)}=n \frac{1}{\sqrt{1-x^{2}}} \operatorname{Im} \mathrm{e}^{i n \arccos (x)} \\
T_{n}^{\prime}(x)=n U_{n-1}(x) \\
U_{n}^{\prime}(x)=\frac{1}{n+1} T_{n+1}^{\prime \prime}(x)
\end{gathered}
$$

Now, by operator calculus we have the identity:

$$
\begin{aligned}
\sin \hat{B} & \equiv \sum_{k=0}^{\infty}(-1)^{k} \frac{B^{2 k+1}}{(2 k+1) !} \equiv:\left(1-\hat{X}^{2}\right)^{\frac{1}{2}} \sum_{k=0}^{\infty}(-1)^{k} \frac{\left(1-\hat{X}^{2}\right)^{k} D_{x}^{2 k}}{(2 k+1) !} D_{x} \\
& \equiv(1-\hat{X})^{\frac{1}{2}} \frac{\sin \hat{B}}{\hat{B}} D_{x}
\end{aligned}
$$

The above identity gives:

$$
\begin{aligned}
\sin \hat{B} x^{n+1}=: & (1-\hat{X})^{\frac{1}{2}} \frac{\sin \hat{B}}{\hat{B}}(n+1) x^{n}=:(1-x)^{\frac{1}{2}} U_{n}(x) \\
& U_{n}(x)=:\left(1-x^{2}\right)^{-\frac{1}{2}} \sin \hat{B} x^{n+1}
\end{aligned}
$$

\subsection{Symbolic Formula for Chebyshev Polynomials of Second Kind}

From the formula (36),

$$
\begin{gathered}
U_{n}(x)=:\left(1-x^{2}\right)^{-\frac{1}{2}} \sin \hat{B} x^{n+1} \\
U_{n}(x)=(n+1) \sum_{k=0}^{\infty}(-1)^{k} \frac{\left(1-x^{2}\right)^{k} D_{x}^{2 k}}{(2 k+1) !} x^{n}
\end{gathered}
$$

and the fact that,

$$
\partial_{x+y} \equiv \partial_{x} \equiv \partial_{y}
$$


we get:

$$
\begin{aligned}
U_{n}(x+y) & =(n+1) \sum_{k=0}^{\infty}(-1)^{k} \frac{\left(1-(x+y)^{2}\right)^{k}}{(2 k+1) !} \partial_{y}^{2 k}(x+y)^{n} \\
& =(n+1) \sum_{k=0}^{\infty}(-1)^{k} \frac{\left(1-(x+y)^{2}\right)^{k}}{(2 k+1) !} \partial_{y}^{2 k} \sum_{l=0}^{n}\left(\begin{array}{l}
n \\
l
\end{array}\right) x^{n-l} y^{l}
\end{aligned}
$$

For $y=0$,

$$
\begin{aligned}
U_{n}(x) & =(n+1) \sum_{k=0}^{\infty}(-1)^{k} \frac{\left(1-x^{2}\right)^{k}}{(2 k+1) !}\left(\begin{array}{c}
n \\
2 k
\end{array}\right)(2 k) ! x^{n-2 k} \\
& =(n+1) \sum_{k=0}^{\left[\frac{n}{2}\right]}(-1)^{k} \frac{\left(1-x^{2}\right)^{k}}{2 k+1}\left(\begin{array}{c}
n \\
2 k
\end{array}\right) x^{n-2 k}
\end{aligned}
$$

so that we get the symbolic formula,

$$
U_{n}(x)=:(n+1)(\Gamma+x)^{n}
$$

where,

$$
\Gamma_{2 k}(x)=\frac{(-1)^{k}\left(1-x^{2}\right)^{k}}{2 k+1}, \quad \Gamma_{2 k+1}(x)=0
$$

with $k$ pair for $U_{n}(x)$ to have the parity of $n$.

Examples:

$$
\begin{gathered}
U_{0}(x)=1 \\
U_{1}(x)=2\left(\Gamma_{0} x\right)=2 x \\
U_{2}(x)=3\left(\Gamma_{0} x^{2}+\Gamma_{2}\right)=3\left(x^{2}+\frac{1}{3}\left(x^{2}-1\right)^{1}\right)=4 x^{2}-1 \\
U_{3}(x)=4\left(x^{3}+\frac{3}{3}\left(x^{2}-1\right) x\right)=8 x^{3}-4 x \\
U_{4}(x)=5\left(\left(x^{4}+6 \frac{1}{3}\left(x^{2}-1\right) x^{2}\right)+\frac{1}{5}\left(x^{2}-1\right)^{2}\right)=16 x^{4}-12 x^{2}+1
\end{gathered}
$$

\section{Remarks and Conclusions}

The principal aim of this work is to propose to researchers and students two formulae having the symbolic form $(C+x)^{n}$ for calculating Chebyshev polynomials. This is possible by utilizing the special operator $\hat{B}_{k} \equiv\left(1-x^{2}\right)^{k} D_{x}^{2 k}$ for defining them and the common property $\partial_{x+y} \equiv \partial_{x} \equiv \partial_{y}$. By the way, we expose the proofs for obtaining more concisely their generating functions as so as a lot but not all of their properties.

The author highly appreciates the invitation of Prof. Dr. Christian Corda, editor in chief of the Journal of High Energy Physics, Gravitation and Cosmology towards him for publication of this work in the Journal. He thanks Ms. Zoey Yang for helping him in realizing the formality of this publication. 


\section{Conflicts of Interest}

The author declares no conflicts of interest regarding the publication of this paper.

\section{References}

[1] Markov, Andreevich, A., Sonin, N. and Oeuvres, I. (1962) Commissionairesde l'Académie impériale des sciences, Chelsea, New York.

[2] Edouard, L. and des nombres, T. (1891) Topics: Number Theory. Paris GauthierVillars, Paris, 296-297.

https://archive.org/details/thoriedesnombr01lucauoft/page/n7/mode/2up

[3] Si, D.T. (2016) Operator Calculus. Edification and Utilization. LAP Lambert Academic Publishing, Omniscriptum GmbH\&Co., Saarbrücken, Germany.

[4] Abramowitz, M. and Stegun, I.A. (1964) Handbook of Mathematical functions with Formulas, Graphs, Mathematical Tables. Washington DC, USA.

[5] Cesarano, C. (1974) Identities and Generating Functions on Chebyshev Polynomials. https://www.uninettunouniversity.net/allegati/1/Identities.pdf

[6] Ayoub, R. (1974) Euler and the Zeta Function. The American Mathematical Monthly, 81, 1067-1086.

[7] Coen, L.E.S. (1996) Sums of Powers and the Bernoulli Numbers. Master's Thesis, Eastern Illinois University, USA. http://thekeep.eiu.edu/theses/1896 